\title{
Spatial Variations and Evolution of PAH, Gas, and Dust Properties in Nearby Major Mergers
}

\author{
S. Haan \\ CSIRO Astronomy \& Space Science, Marsfield NSW 2122, Australia \\ email: sebastian.haan@csiro.au
}

\begin{abstract}
Interactions and mergers of gas-rich galaxies are important drivers of galaxy evolution, in particular in reshaping spirals and fueling powerful starbursts and supermassive nuclear black holes. Hubble Space Telescope observations of nuclei in merging galaxies have shown an increasing nuclear luminosity density in the optical/near-infrared light with advancing merger stage (Laine et al. 2003, Rossa et al. 2007, Veilleux et al. 2009, Haan et al. 2011a), suggesting an increase of nuclear starburst activity. To address the physical conditions in major mergers and excitation mechanisms such as shocks and starbursts, it is necessary to resolve the spatial variations of the warm dust, molecular gas, and ionized gas on subgalactic scales. Here we summarize the main results based on Spitzer IRS spectral mapping observations of a sample of eight nearby, IR-bright galaxies of the Toomre Sequence (Toomre \& Toomre 1972) that span the range from early through mid- to late-stage major mergers (Haan et al. 2011b).

We find that the cold molecular gas component as observed in the CO emission line does not correlate with the warm gas in early merger systems, leading to large spatial variations in the ratio of cold-to-warm molecular gas. In particular, the cold molecular gas distribution in NGC 4676 and NGC 6621 show a significant offset not only from the warm gas component, but also from the stellar (NIR) and dust distribution (PAH, mid-IR continuum). On locally resolved scales within a merger, we find an intrinsic relation between $\mathrm{H}_{2}$ and $\mathrm{PAH}$ flux as given by a power law with a very similar exponent $(0.61 \pm 0.05)$ for all galaxies but with a constant that can vary up to a factor of ten between different galaxies. These findings suggest a common dominant excitation mechanism for $\mathrm{H}_{2}$ emission over a large range of global $\mathrm{H}_{2} / \mathrm{PAH}$ flux ratios in major mergers. The spatial extent of the warm gas component $\left(\mathrm{H}_{2}\right)$ is larger than for the $\mathrm{PAH}$ emission (in all galaxies but more extrem in mid-stage merger), which leads to an increase of the $\mathrm{H}_{2} / \mathrm{PAH}$ ratio as function of radius. Two merger systems (NGC 7592 and NGC 6621) show evidence for buried star formation in overlap regions between the nuclei, namely strong $\mathrm{PAH},[\mathrm{Ne} \mathrm{II}],[\mathrm{Ne} \mathrm{III}]$ and warm gas $\left(\mathrm{H}_{2}\right)$ emission, but no apparent corresponding $\mathrm{CO}(\mathrm{J}=1-0)$ emission. Our findings also demonstrate that the variations of the physical conditions within a merger are much larger than any systematic trends along the Toomre Sequence.
\end{abstract}

Keywords. galaxies: ISM — galaxies: interactions — galaxies: starburst

\section{References}

Haan, S., Surace, J. A., Armus, L., et al. 2011a, AJ, 141, 100

Haan, S., Armus, L., Laine, S., et al. 2011b, ApJS, 197, 27

Laine, S., van der Marel, R. P., Rossa, J., Hibbard, J. E., Mihos, J. C., Böker, T., \& Zabludoff, A. I. $2003, A J, 126,2717$

Rossa, J., Laine, S., van der Marel, R. P., Mihos, J. C., Hibbard, J. E., Böker, T., \& Zabludoff, A. I. $2007, A J, 134,2124$

Toomre, A. \& Toomre, J. 1972, ApJ, 178, 623

Veilleux, S., et al. 2009, ApJ, 701, 587 